BMJ Open

Sport \&

Exercise

Medicine

\section{Content reporting of exercise interventions in rotator cuff disease trials: results from application of the Consensus on Exercise Reporting Template (CERT)}

To cite: Major DH, Røe Y, Grotle $\mathrm{M}$, et al. Content reporting of exercise interventions in rotator cuff disease trials: results from application of the Consensus on Exercise Reporting Template (CERT). BMJ Open Sport \& Exercise Medicine 2019;5:e000656. doi:10.1136/ bmjsem-2019-000656

Accepted 3 December 2019

Check for updates

C) Author(s) (or their employer(s)) 2019. Re-use permitted under CC BY. Published by BMJ.

${ }^{1}$ Faculty of Health Sciences, Oslo Metropolitan University, 0slo, Norway

${ }^{2}$ Research and Communication Unit for Musculoskeletal Health, Oslo University Hospital, Oslo, Norway

${ }^{3}$ Cabrini Institute, Monash Department of Clinical Epidemiology, Melbourne, Victoria, Australia

${ }^{4}$ Monash University Department of Epidemiology and Preventive Medicine, Melbourne, Victoria, Australia

Correspondence to Dr Daniel H Major; danima@oslomet.no

\section{ABSTRACT \\ Background Exercise interventions are frequently} recommended for patients with rotator cuff disease, but poor content reporting in clinical trials of exercise limits interpretation and replication of trials and clinicians' ability to deliver effective exercise protocols. The Consensus on Exercise Reporting Template (CERT) was developed to address this problem.

Objective To assess completeness of content reporting of exercise interventions in randomised controlled trials for patients with rotator cuff disease and the inter-rater reliability of the CERT.

Design Critical appraisal.

Methods Independent pairs of reviewers applied the CERT to all 34 exercise trials from the most recent Cochrane Review evaluating the effect of manual therapy and exercise for patients with rotator cuff disease. We used the CERT Explanation and Elaboration Statement to guide assessment of whether each of the 19-item criteria were clearly described (score 0-19; higher scores indicate better reporting). Percentage agreement and the prevalence and bias adjusted kappa (PABAK) coefficient were used to measure inter-rater reliability.

Results The median CERT score was 5 (range 0-16). Percentage agreement was high for 15 items and acceptable for 4 items. The PABAK coefficient indicated excellent (5 items), substantial (11 items) and moderate (3 items) inter-rater agreement.

Conclusion The description of exercise interventions for patients with rotator cuff disease in published trials is poorly reported. Overall, the inter-rater reliability of the CERT is high/acceptable. We strongly encourage journals to mandate use of the CERT for papers reporting trial protocols and results investigating exercise interventions.

\section{INTRODUCTION}

Shoulder pain is a prevalent and often long-lasting complaint. ${ }^{2}$ Presentation for shoulder pain has an incidence of 10 per 1000 in primary care $^{34}$ and prevalence of $7 \%-26 \% .^{2}$ People with shoulder disorders report experiencing pain, physical function/

\section{Recommendations for future research}

Evaluate the effect of journal implementation of the Consensus on Exercise Reporting Template (CERT) reporting guideline on the quality of the content reporting of exercise interventions.

Determine which CERT items are/are not essential to intervention success in trials investigating the value of exercise.

- Further refinement and evaluation of use of the CERT to better develop and describe exercise interventions and their important components.

What is already known?

- Exercise interventions are recommended as a firstline treatment for people with rotator cuff disease although there is low-quality evidence that exercise interventions may provide limited benefits.

- The content reporting of complex interventions, such as exercise interventions, are often poorly reported, which limits interpretation and replication of trials and clinicians' ability to deliver effective exercise protocols where they exist.

- Early studies have demonstrated that the CERT, developed to address the problem of incomplete reporting of exercise interventions, may be of value for evaluating exercise interventions in musculoskeletal trials.

\section{What are the new findings?}

- The completeness of content reporting of exercise intervention in randomised controlled trials of trials for people with rotator cuff disease is poor.

- The CERT is a reliable tool to evaluate the completeness of reporting of exercise interventions in trials.

activity limitations, participation restriction, sleep disruption, cognitive dysfunction, emotional distress and other pathophysiological manifestations (other than pain). ${ }^{5}$ The 
most common subgroup of shoulder pain is rotator cuff disease, ${ }^{36}$ an umbrella term for various disorders of the rotator cuff, such as subacromial impingement syndrome, rotator cuff tendonitis or tears, and subacromial bursitis. Exercise interventions have been recommended as a first-line treatment for patients with rotator cuff disease. ${ }^{7}$ However, systematic reviews have reported conflicting conclusions about their effectiveness in reducing pain and disability. ${ }^{8-11}$

We know from previous studies that where description of interventions are available, they seldom provide the level of detail required for other researchers to validate trials through replication. ${ }^{12-18}$ Inadequate content reporting of interventions also limits the clinician's ability to interpret the study findings and to deliver an effective exercise protocol in their clinical practice, ${ }^{19}$ and means it may not be possible to determine which specific components of exercise interventions may be associated with better (or worse) outcomes. ${ }^{20}{ }^{21}$ So far, no studies have been conducted to specifically assess the content reporting of exercise interventions investigated in clinical trials for patients with rotator cuff disease.

In order to improve the content reporting of interventions, the Template for Intervention Description and Replication (TIDieR) ${ }^{22}$ checklist was developed. However, this guide was not specifically designed for exercise interventions and does not cover all important exercise prescription domains. The Consensus on Exercise Reporting Template (CERT) was developed to specifically address the problem of incomplete reporting of exercise interventions. ${ }^{23}{ }^{24}$ Based on evidence from a systematic review ${ }^{12}$ and subsequent international Delphi consensus study that included 137 experts, it can be used to both review existing published trials of exercise interventions and act as a template when designing and evaluating exercise interventions. ${ }^{24}$

The main objective of this study was to assess the content reporting of exercise interventions in randomised controlled trials (RCTs) for patients with rotator cuff disease by applying the CERT. The secondary objective was to assess the inter-rater reliability of the 19 CERT items.

\section{METHODS}

\section{Eligibility criteria}

We used the recently published 2016 Cochrane Review evaluating the effect of manual therapy and exercise for reducing pain and improving function for patients with rotator cuff disease to identify RCTs for inclusion in this study. ${ }^{11}$ Page et $a l^{11}$ included RCTs that compared exercise to placebo, no treatment, usual care or another active intervention among adults ( $\geq 18$ years) with rotator cuff disease. The term 'rotator cuff disease' was used in the review for disorders of the rotator cuff labelled and/or defined by the trial authors using terminology such as subacromial impingement syndrome, rotator cuff tendonitis or tendinopathy, supraspinatus, infraspinatus or subscapularis tendonitis, subacromial bursitis or rotator cuff tears. Trials could include interventions provided to participants in any setting (eg, outpatient, at home or in the community) and must have involved the prescription of a supervised or unsupervised exercise programme. The intervention could have been with or without the addition of other components (eg, manipulation, lifestyle modification or counselling).

We included 34 exercise trials reported up to March 2015 from Page $e t a l$ 's Cochrane Review. ${ }^{11}$

\section{Data extraction guidelines}

We used previously described data extraction guidelines to standardise the information that was extracted from each included paper. ${ }^{23}$ Descriptive data were systematically extracted into a spreadsheet, checked for consistency and merged into one document. In order to ensure a similar understanding of the application of the CERT across five reviewers, all reviewers independently pilot tested the data extraction form using one study, which was not included in the final 34 reviewed. All reviewers discussed their CERT ratings on a video conference in pairs with DHM. We estimated the time of the familiarisation process to be approximately 1.5 hours.

\section{Application of the CERT}

Two reviewers independently scored each included study by applying the CERT. ${ }^{23}$ Five reviewers were involved in the application of the CERT (CF, RLJ, YR, MG and DHM). Three reviewers (CF, RLJ and MG) applied the CERT in five trials each; one reviewer (YR) applied the CERT in 19 trials; and another reviewer (DHM) applied the CERT in all included trials. The CERT includes 16 categories and 19 separate items considered essential in the reporting of reproducible exercise interventions listed under seven domains: what (materials), who (provider), how (delivery), where (location), when and how much (dosage), tailoring (what and how) and how well (compliance/planned and actual). ${ }^{23}$ The CERT domains include information about any equipment used for exercises, the exercise instructor, core procedural and contextual elements of the exercise intervention that are required for replication, information about participant motivation strategies and whether, and how well, participants complied with the exercise programme.

A more detailed description of the CERT items is available in the Explanation and Elaboration Statement. ${ }^{23}$ This statement was used to guide the scope and interpretation of each CERT item. Each CERT item was rated as 'yes' (criterion met, indicating item clearly reported), 'no' (indicating item not reported or not clearly described) or 'unsure', and an overall rating of the exercise description was also made. For no or unsure responses, detailed comments about what was missing or what was unclear were recorded. We summed the number of items rated as yes to compute a total score ranging from 0 to $19(0=$ no items clearly described to $19=$ all CERT items clearly described). 
Table 1 Description of the included studies

\begin{tabular}{|c|c|c|c|c|}
\hline $\begin{array}{l}\text { First author, } \\
\text { year }\end{array}$ & Country & Main components of the exercise intervention & $\begin{array}{l}\text { Open } \\
\text { access }\end{array}$ & $\begin{array}{l}\text { Needed supplementary } \\
\text { material }\end{array}$ \\
\hline $\begin{array}{l}\text { Ainsworth, } \\
2009\end{array}$ & England & $\begin{array}{l}\text { Stretching exercises, strengthening exercises, posture correction and } \\
\text { adaptation of functional activities }\end{array}$ & Yes $^{45}$ & No \\
\hline Bae, 2011 & South Korea & Motor control and strengthening exercises & Yes $^{37}$ & No \\
\hline Baskurt, 2011 & Turkey & $\begin{array}{l}\text { Standardised flexibility, strengthening and Codman exercises; group } \\
\text { II additionally performed scapular stabilisation exercise }\end{array}$ & $\mathrm{No}^{38}$ & No \\
\hline $\begin{array}{l}\text { Beaudreuil, } \\
2011\end{array}$ & France & Dynamic humeral centring aimed at lowering the humeral head & $\mathrm{No}^{35}$ & $\begin{array}{l}\text { Online appendix (open } \\
\text { access) }\end{array}$ \\
\hline Bennell, 2010 & Australia & $\begin{array}{l}\text { Improving dynamic scapular control, strengthening scapular } \\
\text { stabiliser and rotator cuff muscles, improving shoulder and thoracic } \\
\text { posture and increasing range of motion of thoracic extension }\end{array}$ & Yes $^{34}$ & $\begin{array}{l}\text { Protocol paper }{ }^{71} \text { and } \\
\text { online appendix (open } \\
\text { access) }\end{array}$ \\
\hline Blume, 2015 & USA & $\begin{array}{l}\text { Eccentric and concentric exercises aiming at optimising rotator cuff } \\
\text { and scapular muscle recruitment }\end{array}$ & Yes $^{42}$ & $\begin{array}{l}\text { Appendix (open } \\
\text { access) }\end{array}$ \\
\hline Brox, 1993 & Norway & $\begin{array}{l}\text { Resistance training of the shoulder rotators and scapular stabilising } \\
\text { muscles }\end{array}$ & Yes $^{39}$ & No \\
\hline Celik, 2009 & Turkey & $\begin{array}{l}\text { Exercises below or above } 90^{\circ}, \text { T-bar (wand) exercises, posterior } \\
\text { capsule stretching and internal rotation exercises and rotator cuff } \\
\text { strengthening exercises were performed. }\end{array}$ & Yes $^{57}$ & No \\
\hline Cloke, 2008 & England & $\begin{array}{l}\text { Kinetic chain exercises, scapular stabilisation, range of motion } \\
\text { exercises, closed chain exercises, plyometric exercises and rotator } \\
\text { cuff exercises }\end{array}$ & $\mathrm{No}^{58}$ & $\begin{array}{l}\text { Kibler } 1998^{31} \\
\text { (not open access) }\end{array}$ \\
\hline Dickens, 2005 & England & $\begin{array}{l}\text { Exercises for the recruitment and strength of scapulothoracic } \\
\text { muscles and rotator cuff }\end{array}$ & $\mathrm{No}^{46}$ & No \\
\hline $\begin{array}{l}\text { Djordjevic, } \\
2012\end{array}$ & Serbia & $\begin{array}{l}\text { Pendulum exercises and pain-limited, active ROM exercises of } \\
\text { shoulder elevation, depression, flexion, abduction, rotations and } \\
\text { strengthening exercises. Strengthening exercises were isometric in } \\
\text { nature, working on the external shoulder rotators, internal rotators, } \\
\text { biceps, deltoid and scapular stabilisers. }\end{array}$ & $\mathrm{No}^{43}$ & No \\
\hline $\begin{array}{l}\text { Engebretsen, } \\
2009\end{array}$ & Norway & $\begin{array}{l}\text { Exercises with a principal focus of relearning of normal movement } \\
\text { patterns, which could then be transferred to daily activities }\end{array}$ & Yes $^{47}$ & $\begin{array}{l}\text { Bøhmer, } 1998^{29} \text { (not } \\
\text { open access) }\end{array}$ \\
\hline Ginn, 2005 & Australia & $\begin{array}{l}\text { Stretches aimed at lengthening shortened shoulder muscles, } \\
\text { exercises aimed at strengthening weakened shoulder muscles, } \\
\text { including improving coordination between muscles, and motor } \\
\text { retraining aimed at restoring scapulohumeral rhythm during the } \\
\text { performance of upper limb tasks }\end{array}$ & Yes $^{48}$ & No \\
\hline Giombini, 2006 & Italy & $\begin{array}{l}\text { Pendular swinging in prone position in flexion and extension of the } \\
\text { shoulder and passive glenohumeral joint stretching exercises }\end{array}$ & $\mathrm{No}^{59}$ & No \\
\hline Haahr, 2005 & Denmark & $\begin{array}{l}\text { Exercises aiming at strengthening the stabilisers and decompressors } \\
\text { of the shoulder }\end{array}$ & Yes $^{49}$ & No \\
\hline Hay, 2003 & England & $\begin{array}{l}\text { Active training of the periscapular muscles and strengthening of the } \\
\text { stabilising muscles of the shoulder joint }\end{array}$ & Yes $^{50}$ & No \\
\hline $\begin{array}{l}\text { Holmgren, } \\
2012\end{array}$ & Sweden & $\begin{array}{l}\text { Strengthening eccentric exercises for the rotator cuff and } \\
\text { strengthening concentric/eccentric exercises for the scapula } \\
\text { stabilisers }\end{array}$ & Yes $^{36}$ & $\begin{array}{l}\text { Online appendix (open } \\
\text { access) }\end{array}$ \\
\hline $\begin{array}{l}\text { Kachingwe, } \\
2008\end{array}$ & USA & $\begin{array}{l}\text { The exercise programme included posterior capsule stretching, } \\
\text { postural correction exercises, and an exercise programme focusing } \\
\text { on rotator cuff strengthening and scapular stabilisation }\end{array}$ & Yes $^{51}$ & No \\
\hline $\begin{array}{l}\text { Littlewood, } \\
2014\end{array}$ & England & Self-managed loaded exercise using a single exercise & Yes $^{52}$ & $\begin{array}{l}\text { Development paper } \\
\text { (open access) }\end{array}$ \\
\hline $\begin{array}{l}\text { Lombardi, } \\
2008\end{array}$ & Brazil & $\begin{array}{l}\text { Strengthening exercises for the shoulder (flexors, extensors, medial } \\
\text { and lateral rotators) }\end{array}$ & Yes $^{53}$ & No \\
\hline Ludewig, 2003 & USA & $\begin{array}{l}\text { Stretching of the pectoralis minor and for the posterior shoulder, } \\
\text { muscle relaxation of the trapezius and strengthening exercises of } \\
\text { external rotators and serratus anterior }\end{array}$ & Yes $^{60}$ & No \\
\hline
\end{tabular}


Table 1 Continued

\begin{tabular}{|c|c|c|c|c|}
\hline $\begin{array}{l}\text { First author, } \\
\text { year }\end{array}$ & Country & Main components of the exercise intervention & $\begin{array}{l}\text { Open } \\
\text { access }\end{array}$ & $\begin{array}{l}\text { Needed supplementary } \\
\text { material }\end{array}$ \\
\hline $\begin{array}{l}\text { Maenhout, } \\
2013\end{array}$ & Belgium & $\begin{array}{l}\text { Internal and external rotation resisted with an elastic band. The } \\
\text { intervention group additionally performed the eccentric phase of } \\
\text { full can (thumb up) abduction in the scapular plane with a dumbbell } \\
\text { weight }\end{array}$ & $\mathrm{No}^{61}$ & No \\
\hline Martins, 2012 & Brazil & $\begin{array}{l}\text { Strengthening and stretching exercises were carried out for } \\
\text { muscles of the shoulder complex with or without the addition of } \\
\text { proprioceptive exercises }\end{array}$ & Yes $^{62}$ & $\begin{array}{l}\text { Kuhn, } 2009^{32} \\
\text { (not open access) }\end{array}$ \\
\hline Marzetti, 2014 & Italy & $\begin{array}{l}\text { Neurocognitive therapeutic exercises with the aim to teach the } \\
\text { patient pathological elements, avoiding compensation and how to } \\
\text { rebuild and recover movements in a smooth and functional way. } \\
\text { Traditional therapeutic exercise contained strengthening exercises } \\
\text { of the rotator cuff and scapular stabilising muscles, stretching } \\
\text { exercises, Codman's pendulum exercises and exercises with elastic } \\
\text { band }\end{array}$ & $\mathrm{Yes}^{40}$ & No \\
\hline $\begin{array}{l}\text { Moosmayer, } \\
2014\end{array}$ & Norway & $\begin{array}{l}\text { Individualised exercise treatment aimed at correction of scapula mal } \\
\text { positioning at rest and the restoration of ideal scapula positioning } \\
\text { and centring of the humeral head during movement }\end{array}$ & Yes $^{54}$ & $\begin{array}{l}\text { A Norwegian } \text { book }^{*} \text { and } \\
\text { an appendix }\end{array}$ \\
\hline Østerås, 2008 & Norway & $\begin{array}{l}\text { A combination of aerobic exercises and low-dosage or high-dosage } \\
\text { medical exercise therapy }\end{array}$ & $\mathrm{No}^{63}$ & No \\
\hline Rhon, 2014 & USA & Exercises directed to the shoulder girdle or thoracic or cervical spine & $\mathrm{No}^{55}$ & $\begin{array}{l}\text { Protocol paper } \\
\text { and appendix (open } \\
\text { access) }\end{array}$ \\
\hline $\begin{array}{l}\text { Şenbursa, } \\
2011\end{array}$ & Turkey & $\begin{array}{l}\text { Range of motion, stretching and strengthening exercises for the } \\
\text { rhomboid, levator scapulae, serratus anterior and rotator cuff } \\
\text { muscles }\end{array}$ & $\mathrm{No}^{56}$ & No \\
\hline Struyf, 2013 & Belgium & $\begin{array}{l}\text { Stretching and motor control training of the scapula } \\
\text { Exercise therapy comprised of an eccentric muscle strength training } \\
\text { programme of the rotator cuff muscles }\end{array}$ & $\mathrm{No}^{41}$ & $\begin{array}{l}\text { Mottram, } 1997^{30} \text { (not } \\
\text { open access) }\end{array}$ \\
\hline Subasi, 2012 & Turkey & $\begin{array}{l}\text { Stretching and range of motion exercises followed by land-based or } \\
\text { water-based strengthening exercises }\end{array}$ & $\mathrm{No}^{64}$ & No \\
\hline $\begin{array}{l}\text { Szczurko, } \\
2009\end{array}$ & Canada & $\begin{array}{l}\text { Isometric shoulder strength training and a series of passive, active- } \\
\text { assisted and active range of motion, muscle strengthening and joint } \\
\text { therapy }\end{array}$ & Yes $^{65}$ & $\begin{array}{l}\text { Hagberg, } 2000^{74} \text { (open } \\
\text { access) } \\
\text { Levoska, } 1993^{33} \text { (not } \\
\text { open access) }\end{array}$ \\
\hline Walther, 2004 & Germany & $\begin{array}{l}\text { Exercises aiming at strengthening the depressor muscles and } \\
\text { centring the humeral head }\end{array}$ & $\mathrm{No}^{66}$ & No \\
\hline Wang, 2006 & USA & $\begin{array}{l}\text { Customised exercises designed by the authors to correct different } \\
\text { shoulder classification systems }\end{array}$ & $\mathrm{No}^{67}$ & No \\
\hline Winters, 1997 & Netherlands & Exercise therapy & $Y_{e s}{ }^{44}$ & No \\
\hline
\end{tabular}

${ }^{*}$ Not used when assessing the content reporting.

If the authors specifically referred to published protocols, online appendices and supplementary data, the reviewers retrieved and extracted these additional data when relevant. The reviewers also recorded whether the study was published in an open access journal and how easy the intervention description was to access (ie, available in the published paper or required additional data from other sources and, if so, whether this was open access).

Following completion of the review by both reviewers, any disagreements were discussed. If agreement could not be reached, an independent arbiter from the research team was to be consulted.

\section{Risk of bias assessment}

Risk of bias assessments of the included trials, based on the Cochrane Risk of Bias Tool, ${ }^{25}$ were taken from the original Cochrane Review. ${ }^{11}$ The following domains were assessed: random sequence generation, allocation concealment, blinding of participants and personnel, and blinding of outcome assessment (subjective and objective). The risk of bias figure was prepared using RevMan V.5.3 (The Nordic Cochrane Centren, Copenhagen)

\section{Inter-rater reliability}

Inter-rater reliability of the CERT was assessed for each of the 19 CERT items (including subitems $a$ and $b$ for 




Figure 1 Risk of bias summary: Cochrane Review of authors' judgements about each risk of bias item for each included study. Empty cells mean that objective outcomes were not measured in the trial, so an assessment of the risk of bias due to lack of blinding of such outcomes was not applicable. items 7,14 and 16) using percentage agreement ${ }^{26}$ and the prevalence and bias adjusted kappa (PABAK) coefficient. ${ }^{27}$ While kappa statistics measures chance-adjusted agreement and is therefore more robust than simple percentage agreement, when the prevalence of one of the categories is much higher than that of the other, chance agreement will be high and kappa can have unexpectedly low values. ${ }^{26-28}$ For percentage agreement, a score of $70 \%$ or greater is considered acceptable and $\geq 80 \%$ is considered high. ${ }^{28}$ For PABAK coefficients, the strength of agreement is interpreted as follows: $0=$ poor, $0.01-0.20=$ slight,$\quad 0.21-0.40=$ fair, $\quad 0.41-0.60=$ moderate, $0.61-0.80=$ substantial and $0.81-1=$ excellent $^{28}$

\section{Data analysis}

Data were entered into SPSS V.22 and were analysed using descriptive statistics and narrative summaries. For each study, the total CERT score was presented together with the percentage of a maximum CERT score of 19 . The bootstrapped median was calculated using STATA (Version 12. College Station, TX, United States of America). Bootstrapping is a statistical method based on simulation of random sampling from the available data. We have performed 10000 repetitions of the sampling creating samples with the same statistical properties as the original data set. The estimate of the median and 95\% CI were calculated directly from the simulated repeated sampling. In this way, we did not have to assume any statistical distribution for the median and achieved a higher level of precision when constructing the CI.

\section{RESULTS}

Twenty of the 34 trials were open access articles (table 1). The trials were from 15 different countries, and the main components of the exercise interventions most often included strengthening, scapula stabilising, stretching and 'corrective' exercises. Twelve trials referred specifically to supplementary material, and five of these were not open access. ${ }^{29-33}$ Of the included trials, three ${ }^{34-36}$ were judged to be at low risk of bias; eight trials ${ }^{37-44}$ were at unclear risk of bias; and 23 trials ${ }^{45-67}$ were at high risk of bias (figure 1).

\section{Final consensus CERT results}

The CERT evaluation of the reporting of the exercise interventions of the 34 included trials is shown in table 2. The median score was 5 (range $0-16$ ) out of a possible score of 19 . Six trials had a CERT score of $\geq 10$, while the remaining $83 \%(18 / 34)$ all scored $<10$, indicating that the majority of the details of the exercise interventions was missing from the trial reports. The bootstrapped median with 10000 bootstrap samples indicated a median CERT score of 5 (95\% CI 3.5 to 6.1 ).

Four CERT items were clearly described by at least $50 \%$ of the trials (figure 2). These included description of any non-exercise component (23 trials), whether the intervention was supervised or not supervised (21 trials), if the intervention included a home programme (17 


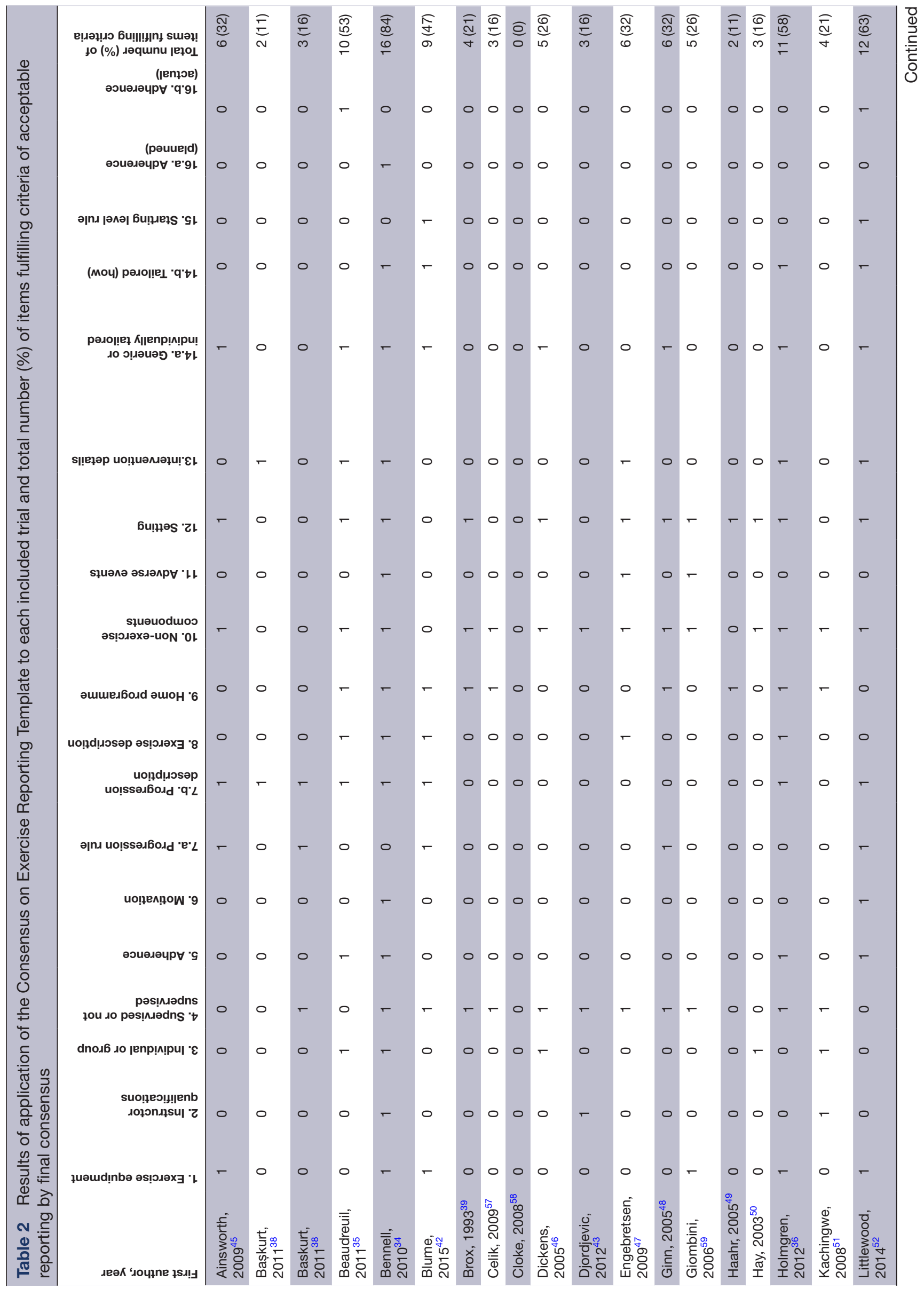









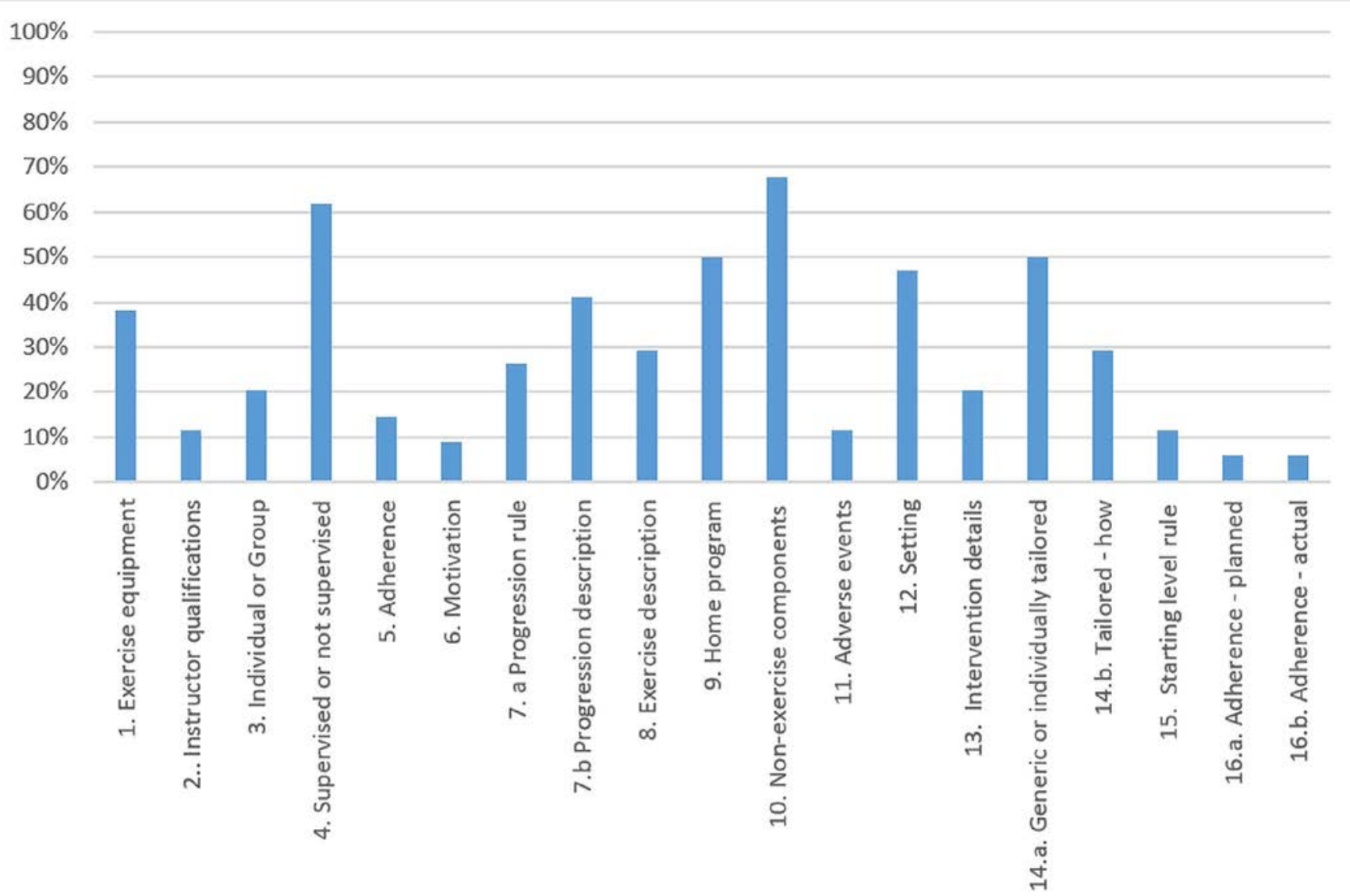

Figure 2 Percentage of interventions (out of 34) with complete reporting for each of the Consensus on Exercise Reporting Template checklist.

trials), and whether it was generic or individually tailored (17 trials). The following items were particularly poorly reported across most trials: motivational strategies were described in only three trials $(9 \%)^{345263}$; how adherence or fidelity was assessed/measured was reported in only two trials $(6 \%)^{3455}$; and to what extent the intervention was delivered as planned was reported in only two trials $(6 \%) .{ }^{35} 52$

\section{Inter-rater agreement of CERT assessment}

Table 3 presents percentage agreement and PABAK coefficients for each CERT item. For a majority of the items, inter-rater agreement was high according to percentage agreement $(15 / 19$ items: $>80 \%)$ and it was substantial (11 items: $0.61-0.80$ ) or excellent (5 items: 0.81-1.0) according to the PABAK. There was acceptable agreement for the descriptions of the type of exercise equipment (item 1) (74\% agreement, PABAK 0.47); qualifications (item 2) (71\% agreement, PABAK 0.41); and which setting the exercises were performed (item 12) $(79 \%$ agreement, PABAK 0.59$)$.

Consensus was reached on all the dissonant items without the need for an independent arbiter. The reason for the disagreements on these items was that one of the reviewers had a stricter interpretation of the CERT Explanation and Elaboration Statement than the other reviewer (items 1, 2 and 9) and reviewer error (item 12). For item 1 (equipment), the authors had described most of the equipment used, but a detailed description was missing for one or more of the exercises. The disagreement on item 2 (qualifications) was because one of the reviewers rated descriptions such as 'experienced physiotherapist', 'highly experienced physiotherapist' and 'musculoskeletal physiotherapist' as clearly described, while the other reviewer had a stricter interpretation of the item and argued that the description of qualification should also include duration of experience. The seven disagreements on item 12 (setting) was caused by reviewer errors, where the setting had been clearly described in the main paper or the protocol, but one of the reviewers had missed it. For the disagreements other than those that were considered errors, the authors chose to agree on the strictest interpretation in all occasions.

\section{DISCUSSION}

The main result of this paper is that trials investigating exercise interventions designed for patients with rotator cuff disease poorly describe the content of their exercise interventions. Most CERT items were not described in enough detail to be able to be replicated, while only four CERT items were clearly described by at least $50 \%$ of the 
Table 3 Percentage agreement and inter-rater reliability for each CERT item

\begin{tabular}{|c|c|c|c|c|}
\hline CERT item & $\begin{array}{l}\% \text { Agreement by } \\
\text { item }^{*}\end{array}$ & Strength of agreement & $\begin{array}{l}\text { PABAK kappa coefficient† } \\
(95 \% \mathrm{Cl})\end{array}$ & $\begin{array}{l}\text { Strength of the } \\
\text { agreement } \neq\end{array}$ \\
\hline 1. Equipment & 74 & Acceptable & $0.47(0.29$ to 0,76$)$ & Moderate \\
\hline 2. Instructor qualifications & 71 & Acceptable & $0.41(0.07$ to 0.76$)$ & Moderate \\
\hline 3. Individual or group & 88 & High & 0.76 (0.48 to 1.0$)$ & Substantial \\
\hline 4. Supervised or not supervised & 88 & High & $0.76(0.51$ to 1.0$)$ & Substantial \\
\hline 5. Adherence & 91 & High & $0.82(0.52$ to 1.0$)$ & Excellent \\
\hline 6. Motivation & 97 & High & $0.94(0.47$ to 1.0$)$ & Excellent \\
\hline 7a. Progression rule & 88 & High & 0.760 .46 to 1.0$)$ & Substantial \\
\hline 7b. Progression description & 88 & High & $0.76(0.48$ to 1.0$)$ & Substantial \\
\hline 8. Exercise description & 91 & High & $0.82(0.54$ to 1.0$)$ & Excellent \\
\hline 9. Home programme & 82 & High & 0.65 (0.38 to 0.92$)$ & Substantial \\
\hline 10. Non-exercise components & 82 & High & 0.65 (0.36 to 0.93$)$ & Substantial \\
\hline 11. Adverse events & 91 & High & $0.82(0.36$ to 1.0$)$ & Excellent \\
\hline 12. Setting & 79 & Acceptable & 0.59 (0.28 to 0.89$)$ & Moderate \\
\hline 13. Intervention details & 85 & High & $0.71(0.38$ to 1.0$)$ & Substantial \\
\hline 14a. Generic or individually tailored & 79 & Acceptable & 0.76 (0.3 to 0.88$)$ & Substantial \\
\hline 14b. Tailored how & 88 & High & $0.76(0.46$ to 1.0$)$ & Substantial \\
\hline 15.Starting level rule & 88 & High & $0.76(0.34$ to 1.0$)$ & Substantial \\
\hline 16a. Adherence (planned) & 88 & High & $0.76(0.7$ to 0.83$)$ & Substantial \\
\hline 16b. Adherence (actual) & 94 & High & 0.88 (0.41 to 1$)$ & Excellent \\
\hline
\end{tabular}

${ }^{*}$ For percentage agreement scores, the strength of agreement is expressed by the following descriptors: $<70 \%=$ poor, $70 \%-79 \%=$ acceptable and $\geq 80 \%=$ high.

†Inter-rater reliability measured using the PABAK coefficient.

fFor PABAK scores, the strength of agreement is expressed by the following descriptors: $0=$ poor, $0.01-0.20=$ slight, $0.21-0.40=$ fair, $0.41-0.60=$ moderate, $0.61-0.80=$ substantial and $0.81-1=$ excellent.

CERT, Consensus on Exercise Reporting Template; PABAK, prevalence and bias adjusted kappa.

included trials. Overall, our results also indicate that the inter-rater reliability of the CERT is high/acceptable.

\section{Comparison with other studies}

Our finding of incomplete descriptions of exercise interventions in our corpus of trials is in keeping with previous studies that have evaluated exercise descriptions using the CERT in trials in knee osteoarthritis and a random sample of musculoskeletal exercise trials. ${ }^{15} 17$ Comparable findings were also found in a systematic review that assessed the content reporting for exercise interventions for patellofemoral pain syndrome using the TIDieR checklist, ${ }^{162}$ and Toigo and Boutellier mechanobiological exercise descriptors. ${ }^{68}$ In contrast to our study, which included a random sample of rotator cuff disease exercise trials, O'Neil et al included only knee osteoarthritis exercise trials that had a Physiotherapy Evidence Database (PEDro) Scale total score of $\geq 6$ out of a maximum of $10{ }^{15}$ The PEDro score evaluates risk of bias as well as adequacy of trial reporting. Based on the CERT scores of each included study reported in O'Neil et al's supplementary material (appendix 1), we calculated that the included trials had an overall median CERT score of 11 (range 4-17). The higher median score may indicate reporting of exercise interventions is better in higher quality trials.
This is also indicated by our results where the three trials judged to be at low risk of bias were among the six trials with a CERT score of $\geq 10$. A post hoc analysis, requested by a reviewer, also revealed a statistically significant $(\mathrm{p}=0.026$ ), weak positive correlation (Spearman's $r=0.38$ ) between the CERT score and the number of low bias items on the Cochrane risk of bias tool. Other possible explanations for the higher CERT score could also be that the content reporting of exercise interventions in trials for people with knee osteoarthritis are generally better than trials for people with rotator cuff disease, or that O'Neil et al were less strict when applying the CERT.

Overall, we determined that the inter-rater reliability of the CERT was high/acceptable by two different agreement measures, and this is keeping with the recently published study by Slade $e t$ al. ${ }^{17}$ However, there are some minor differences across studies for some specific items. We found acceptable/moderate agreement for the CERT item concerning qualifications (item 2), whereas Slade et $a l^{17}$ reported high/excellent inter-rater reliability for this item. This may have been due to lack of clarity that duration of experience is an important consideration, and this needs to be made explicit in the CERT. Slade et $a l^{17}$ reported poor/fair inter-rater reliability for item 
14b (tailored-how) and item 15 (starting rule), whereas we reported high/substantial inter-rater reliability for the same items. The better reliability of these latter items in our study is likely explained by the clarification of how these items should be assessed following the study by Slade et $a l .{ }^{17}$

\section{Strengths and limitations}

A strength of our study was the use of an internationally endorsed reporting guideline for assessing the completeness of descriptions of exercise interventions in clinical trials. The CERT has previously been shown to be user-friendly and time-efficient for review purposes. ${ }^{17}$ In order to ensure similar interpretation of the CERT items, we pilot tested the extraction form for familiarisation purposes before assessing the included trials. We assessed a sample of rotator cuff disease trials investigating the value of exercise from a recent Cochrane Review. This ensured that the included studies were relevant and important for the shoulder research field. Since all the included studies were published prior to the CERT or TIDier, this study reflects the practice of content reporting of exercise interventions without any influence of the new intervention reporting recommendations. Finally, the inter-rater agreement may also have been overestimated due to the small sample size of 34 and the precision of the PABAK estimates, which might have been higher with more studies.

\section{Implications for practice}

To be able to interpret, use, or replicate the research, published reports need to include a sufficiently clear, complete and accurate description of the intervention. ${ }^{18}$ If clinicians, patients and policy makers are to implement the latest evidence from systematic reviews and/or trials, they need to be able to access clearly described interventions that include all the necessary detail about the specific components of an exercise programme. Our results indicate that only 2 of 34 trials reported whether the intervention was delivered as planned, and only 5 trials reported how they assessed/ measured adherence. Not reporting these items hinders accurate interpretation of trial findings, as the reader does not know if the patients/therapists did more/less than what was described in the intervention and/or if the participants adhered to the exercise intervention. Incomplete reporting is also challenging for metaanalysis, which may lead to inappropriate pooling of data from heterogeneous interventions and, in worst case scenarios, erroneous conclusions.

\section{Implications for research}

Detailed description of each CERT item would enable researchers to conduct further research in order to identify which items are (or are not) significantly associated with the outcome (eg, Does the qualification of the instructor matter?). This could inform future exercise interventions. ${ }^{69}$ Trial authors should be obliged to provide a detailed description of their intervention either in the primary paper reporting their results or in a protocol paper. Where further details are provided elsewhere, it is important that these are available via open access and that links remain unbroken over time. The use of the CERT has the potential to advance this field for researchers, peer reviewers, policymakers and clinicians by facilitating an unambiguous description of exercise programmes, which would ultimately lead to less research waste and more timely uptake of evidence into practice. ${ }^{70}$

\section{CONCLUSION}

The completeness of content reporting of exercise interventions in RCTs of trials for people with rotator cuff disease is poor. The CERT is a reliable tool to evaluate the completeness of reporting of exercise interventions in trials. We strongly encourage journals to mandate the use of the CERT when reporting the protocols and results of trials investigating exercise interventions.

Twitter Daniel H Major @danielhmajor

Acknowledgements RB is supported by an Australian National Health and Medical Research Council Senior Principal Research Fellowship.

Contributors RB and DHM conceived the study and all authors contributed to its design and content. DHM, YR, MG, RLJ and CF conducted the review of the included studies. DHM and MCS conducted the data analysis. DHM wrote the first draft of the article. All authors contributed to the revisions of the article. All authors have read and approved the final manuscript.

Funding The authors have not declared a specific grant for this research from any funding agency in the public, commercial or not-for-profit sectors.

Competing interests RB was the senior investigator and guarantor for the development of the CERT.

Patient consent for publication Not required.

Provenance and peer review Not commissioned; externally peer reviewed.

Data availability statement Data are available upon reasonable request.

Open access This is an open access article distributed in accordance with the Creative Commons Attribution 4.0 Unported (CC BY 4.0) license, which permits others to copy, redistribute, remix, transform and build upon this work for any purpose, provided the original work is properly cited, a link to the licence is given, and indication of whether changes were made. See: https://creativecommons.org/ licenses/by/4.0/.

ORCID iD

Daniel H Major http://orcid.org/0000-0001-5204-162X

\section{REFERENCES}

1 Croft P, Pope D, Silman A. The clinical course of shoulder pain: prospective cohort study in primary care. primary care rheumatology Society shoulder Study Group. BMJ 1996;313:601-2.

2 Luime JJ, Koes BW, Hendriksen IJM, et al. Prevalence and incidence of shoulder pain in the general population; a systematic review. Scand J Rheumatol 2004;33:73-81.

3 Ostör AJK, Richards CA, Prevost AT, et al. Diagnosis and relation to general health of shoulder disorders presenting to primary care. Rheumatology 2005;44:800-5.

4 van der Windt DA, Koes BW, de Jong BA, et al. Shoulder disorders in general practice: incidence, patient characteristics, and management. Ann Rheum Dis 1995;54:959-64.

5 Page MJ, O'Connor DA, Malek M, et al. Patients' experience of shoulder disorders: a systematic review of qualitative studies for the OMERACT Shoulder Core Domain Set. Rheumatology 2019;58:1410-21.

6 Linsell L, Dawson J, Zondervan K, et al. Prevalence and incidence of adults consulting for shoulder conditions in UK primary care; patterns of diagnosis and referral. Rheumatology 2006;45:215-21. 
7 Haik MN, Alburquerque-Sendín F, Moreira RFC, et al. Effectiveness of physical therapy treatment of clearly defined subacromial pain: a systematic review of randomised controlled trials. Br J Sports Med 2016;50:1124-34.

8 Littlewood C, Ashton J, Chance-Larsen K, et al. Exercise for rotator cuff tendinopathy: a systematic review. Physiotherapy 2012;98:101-9.

9 Steuri R, Sattelmayer M, Elsig S, et al. Effectiveness of conservative interventions including exercise, manual therapy and medical management in adults with shoulder impingement: a systematic review and meta-analysis of RCTs. Br J Sports Med 2017;51:1340-7.

10 Saltychev M, Äärimaa V, Virolainen P, et al. Conservative treatment or surgery for shoulder impingement: systematic review and metaanalysis. Disabil Rehabil 2015;37:1-8.

11 Page MJ, Green S, McBain B, et al. Manual therapy and exercise for rotator cuff disease. Cochrane Database Syst Rev 2016;1.

12 Slade SC, Keating JL. Exercise prescription: a case for standardised reporting. Br J Sports Med 2012;46:1110-3.

13 Hoffmann TC, Erueti C, Glasziou PP. Poor description of nonpharmacological interventions: analysis of consecutive sample of randomised trials. BMJ 2013;347.

14 Abell B, Glasziou P, Hoffmann T. Reporting and replicating trials of exercise-based cardiac rehabilitation: do we know what the researchers actually did? Circ Cardiovasc Qual Outcomes 2015;8:187-94.

15 O'Neil J, McEwen D, Del Bel MJ, et al. Assessment of the content reporting for therapeutic exercise interventions among existing randomized controlled trials on knee osteoarthritis. Clin Rehabil 2018;32:980-4.

16 Holden S, Rathleff MS, Jensen MB, et al. How can we implement exercise therapy for patellofemoral pain if we don't know what was prescribed? A systematic review. Br J Sports Med 2018;52:385.

17 Slade SC, Finnegan S, Dionne CE, et al. The consensus on exercise reporting template (CERT) applied to exercise interventions in musculoskeletal trials demonstrated good rater agreement and incomplete reporting. J Clin Epidemiol 2018;103:120-30.

18 Glasziou P, Altman DG, Bossuyt P, et al. Reducing waste from incomplete or unusable reports of biomedical research. The Lancet 2014;383:267-76.

19 Glasziou P, Meats E, Heneghan C, et al. What is missing from descriptions of treatment in trials and reviews? BM 2008;336:1472-4.

20 Hanratty CE, McVeigh JG, Kerr DP, et al. The effectiveness of physiotherapy exercises in subacromial impingement syndrome: a systematic review and meta-analysis. Semin Arthritis Rheum 2012;42:297-316.

21 Littlewood C, Malliaras P, Chance-Larsen K. Therapeutic exercise for rotator cuff tendinopathy: a systematic review of contextual factors and prescription parameters. Int J Rehabil Res 2015;38:95-106.

22 Hoffmann TC, Glasziou PP, Boutron I, et al. Better reporting of interventions: template for intervention description and replication (TIDieR) checklist and guide. BMJ 2014;348:g1687.

23 Slade SC, Dionne CE, Underwood M, et al. Consensus on exercise reporting template (CERT): explanation and elaboration statement. Br J Sports Med 2016;50:1428-37.

24 Slade SC, Dionne CE, Underwood M, et al. Consensus on exercise reporting template (CERT): modified Delphi study. Phys Ther 2016;96:1514-24.

25 Higgins JPT, Altman DG, Gøtzsche PC, et al. The Cochrane collaboration's tool for assessing risk of bias in randomised trials. BMJ 2011;343:d5928

26 de Vet HCW, Terwee CB, Knol DL, et al. When to use agreement versus reliability measures. J Clin Epidemiol 2006;59:1033-9.

27 Chen G, Faris P, Hemmelgarn B, et al. Measuring agreement of administrative data with chart data using prevalence unadjusted and adjusted kappa. BMC Med Res Methodol 2009;9:5.

28 Landis JR, Koch GG. The measurement of observer agreement for categorical data. Biometrics 1977;33:159-74.

29 Bøhmer AS, Staff PH, Brox JI. Supervised exercises in relation to rotator cuff disease (impingement syndrome stages II and III): a treatment regimen and its rationale. Physiother Theory Pract 1998;14:93-105.

30 Mottram SL. Dynamic stability of the scapula. Man Ther 1997;2:123-31.

31 Kibler WBEN. Shoulder rehabilitation: principles and practice. Med Sci in Sports Exerc 1998;30:40-50.

32 Kuhn JE. Exercise in the treatment of rotator cuff impingement: a systematic review and a synthesized evidence-based rehabilitation protocol. J Shoulder Elbow Surg 2009;18:138-60.

33 Levoska S, Keinänen-Kiukaanniemi S. Active or passive physiotherapy for occupational cervicobrachial disorders? A comparison of two treatment methods with a 1-year follow-up. Arch Phys Med Rehabil 1993;74:425-30.

34 Bennell K, Wee E, Coburn S, et al. Efficacy of standardised manual therapy and home exercise programme for chronic rotator cuff disease: randomised placebo controlled trial. BMJ 2010;340:c2756.

35 Beaudreuil J, Lasbleiz S, Richette P, et al. Assessment of dynamic humeral centering in shoulder pain with impingement syndrome: a randomised clinical trial. Ann Rheum Dis 2011;70:1613-8.

36 Holmgren T, Björnsson Hallgren H, Öberg B, et al. Effect of specific exercise strategy on need for surgery in patients with subacromial impingement syndrome: randomised controlled study. BMJ 2012;344.

37 Bae YH, Lee GC, Shin WS, et al. Effect of motor control and strengthening exercises on pain, function, strength and the range of motion of patients with shoulder impingement syndrome. J Phys Ther Sci 2011;23:687-92.

38 Bașkurt Z, Bașkurt F, Gelecek N, et al. The effectiveness of scapular stabilization exercise in the patients with subacromial impingement syndrome. J Back Musculoskelet Rehabil 2011;24:173-9.

39 Brox JI, Staff PH, Ljunggren AE, et al. Arthroscopic surgery compared with supervised exercises in patients with rotator cuff disease (stage II impingement syndrome). BMJ 1993;307:899-903.

40 Marzetti E, Rabini A, Piccinini G, et al. Neurocognitive therapeutic exercise improves pain and function in patients with shoulder impingement syndrome: a single-blind randomized controlled clinical trial. Eur J Phys Rehabil Med 2014;50:255-64.

41 Struyf F, Nijs J, Mollekens S, et al. Scapular-focused treatment in patients with shoulder impingement syndrome: a randomized clinical trial. Clin Rheumatol 2013;32:73-85.

42 Blume C, Wang-Price S, Trudelle-Jackson E, et al. Comparison of eccentric and concentric exercise interventions in adults with subacromial impingement syndrome. Int $J$ Sports Phys Ther 2015:10:441-55.

43 Djordjevic OC, Vukicevic D, Katunac L, et al. Mobilization with movement and kinesiotaping compared with a supervised exercise program for painful shoulder: results of a clinical trial. J Manipulative Physiol Ther 2012;35:454-63.

44 Winters JC, Sobel JS, Groenier KH, et al. Comparison of physiotherapy, manipulation, and corticosteroid injection for treating shoulder complaints in general practice: randomised, single blind study. BMJ 1997;314:1320-5.

45 Ainsworth R, Lewis J, Conboy V. A prospective randomized placebo controlled clinical trial of a rehabilitation programme for patients with a diagnosis of massive rotator cuff tears of the shoulder. Shoulder Elbow 2009;1:55-60.

46 Dickens VA, Williams JL, Bhamra MS. Role of physiotherapy in the treatment of subacromial impingement syndrome: a prospective study. Physiotherapy 2005;91:159-64.

47 Engebretsen K, Grotle M, Bautz-Holter E, et al. Radial extracorporeal shockwave treatment compared with supervised exercises in patients with subacromial pain syndrome: single blind randomised study. BMJ 2009;339:b3360.

48 Ginn KA, Cohen ML. Exercise therapy for shoulder pain aimed at restoring neuromuscular control: a randomized comparative clinical trial. J Rehabil Med 2005;37:115-22.

49 Haahr JP, Østergaard S, Dalsgaard J, et al. Exercises versus arthroscopic decompression in patients with subacromial impingement: a randomised, controlled study in 90 cases with a one year follow up. Ann Rheum Dis 2005;64:760-4.

50 Hay EM, Thomas E, Paterson SM, et al. A pragmatic randomised controlled trial of local corticosteroid injection and physiotherapy for the treatment of new episodes of unilateral shoulder pain in primary care. Ann Rheum Dis 2003;62:394-9.

51 Kachingwe AF, Phillips B, Sletten E, et al. Comparison of manual therapy techniques with therapeutic exercise in the treatment of shoulder impingement: a randomized controlled pilot clinical trial. $J$ Man Manip Ther 2008;16:238-47.

52 Littlewood C, Malliaras P, Mawson S, et al. Self-managed loaded exercise versus usual physiotherapy treatment for rotator cuff tendinopathy: a pilot randomised controlled trial. Physiotherapy 2014;100:54-60.

53 Lombardi I, Magri AG, Fleury AM, et al. Progressive resistance training in patients with shoulder impingement syndrome: a randomized controlled trial. Arthritis Rheum 2008;59:615-22.

54 Moosmayer S, Lund G, Seljom US, et al. Tendon repair compared with physiotherapy in the treatment of rotator cuff tears: a randomized controlled study in 103 cases with a five-year follow-up. J Bone Joint Surg Am 2014;96:1504-14.

55 Rhon DI, Boyles RB, Cleland JA. One-Year outcome of subacromial corticosteroid injection compared with manual physical therapy for the management of the unilateral shoulder impingement 
syndrome: a pragmatic randomized trial. Ann Intern Med 2014:161:161-9.

56 Şenbursa G, Baltaci G, Atay Ö Ahmet. The effectiveness of manual therapy in supraspinatus tendinopathy. Acta Orthop Traumatol Turc 2011;45:162-7.

57 Celik D, Akyüz G, Yeldan I. [Comparison of the effects of two different exercise programs on pain in subacromial impingement syndrome]. Acta Orthop Traumatol Turc 2009;43:504-9.

58 Cloke DJ, Watson H, Purdy S, et al. A pilot randomized, controlled trial of treatment for painful Arc of the shoulder. J Shoulder Elbow Surg 2008;17:S17-21.

59 Giombini A, Di Cesare A, Safran MR, et al. Short-Term effectiveness of hyperthermia for supraspinatus tendinopathy in athletes: a short-term randomized controlled study. Am J Sports Med 2006;34:1247-53.

60 Ludewig PM, Borstad JD. Effects of a home exercise programme on shoulder pain and functional status in construction workers. Occup Environ Med 2003;60:841-9.

61 Maenhout AG, Mahieu NN, De Muynck M, et al. Does adding heavy load eccentric training to rehabilitation of patients with unilateral subacromial impingement result in better outcome? A randomized, clinical trial. Knee Surg Sports Traumatol Arthrosc 2013;21:1158-67.

62 Martins LV, Marziale MHP. Assessment of proprioceptive exercises in the treatment of rotator cuff disorders in nursing professionals: a randomized controlled clinical trial. Rev Bras Fisioter 2012;16:502-9.

63 Østerås H, Arild Torstensen T, Arntzen G, et al. A comparison of work absence periods and the associated costs for two different modes of exercise therapies for patients with longstanding subacromial pain. $J$ Med Econ 2008;11:371-81.

64 Subasi V, Toktas H, DÛ S, et al. Water-Based versus land-based exercise program for the management of shoulder impingement syndrome. Turk J Phys Med Rehabil 2012;58:79-84.

65 Szczurko O, Cooley K, Mills EJ, et al. Naturopathic treatment of rotator cuff tendinitis among Canadian postal workers: a randomized controlled trial. Arthritis Rheum 2009;61:1037-45.
66 Walther M, Werner A, Stahlschmidt T, et al. The subacromial impingement syndrome of the shoulder treated by conventional physiotherapy, self-training, and a shoulder brace: results of a prospective, randomized study. J Shoulder Elbow Surg 2004;13:417-23.

67 Wang SS, Trudelle-Jackson EJ. Comparison of customized versus standard exercises in rehabilitation of shoulder disorders. Clin Rehabil 2006;20:675-85.

68 Toigo M, Boutellier U. New fundamental resistance exercise determinants of molecular and cellular muscle adaptations. Eur $J$ Appl Physiol 2006;97:643-63.

69 Hoffmann TC, Oxman AD, loannidis JP, et al. Enhancing the usability of systematic reviews by improving the consideration and description of interventions. BMJ 2017;358.

70 Kent P, O'Sullivan PB, Keating J, et al. Evidence-Based exercise prescription is facilitated by the consensus on exercise reporting template (CERT). Br J Sports Med 2018;52:147-8.

71 Bennell K, Coburn S, Wee E, et al. Efficacy and cost-effectiveness of a physiotherapy program for chronic rotator cuff pathology: a protocol for a randomised, double-blind, placebo-controlled trial. BMC Musculoskelet Disord 2007;8:86.

72 Littlewood C, Malliaras P, Mawson S, et al. Development of a selfmanaged loaded exercise programme for rotator cuff tendinopathy. Physiotherapy 2013;99:358-62.

73 Rhon DI, Boyles RE, Cleland JA, et al. A manual physical therapy approach versus subacromial corticosteroid injection for treatment of shoulder impingement syndrome: a protocol for a randomised clinical trial. BMJ Open 2011;1:e000137.

74 Hagberg M, Harms-Ringdahl K, Nisell R, et al. Rehabilitation of neck-shoulder pain in women industrial workers: a randomized trial comparing isometric shoulder endurance training with isometric shoulder strength training. Arch Phys Med Rehabil 2000;81:1051-8. 